\title{
Nouvelles données sur la flore endémique du sous-secteur de l'Atlas tellien Oranais “O3” (Algérie occidentale)
}

\author{
Mohamed Djamel Miara', Mohammed Ait Hammou', Walid Dahmani' ${ }^{1}$, Moahmed Negadi ${ }^{2}$ et Abdelmadjid \\ Djellaoui ${ }^{1}$ \\ 1 Département de Biologie. Faculté SNV. Université Ibn Khaldoun, Tiaret. 14000. DZ \\ Département de Biologie. Université de Bordj Bouareridj. 34000. DZ
}

\section{Correspondencia}

M. D. Miara

e-mail: miara14130@yahoo.fr

Recibido: 20 marzo 2018

Aceptado: 18 septiembre 2018

Publicado on-line: noviembre 2018

\begin{abstract}
Résumé
Nouvelles données sur la flore endémique du sous-secteur de l'Atlas tellien Oranais «O3» (Algérie occidentale). Cette étude est une première synthèse régionale visant à proposer une nouvelle liste de la flore endémique du soussecteur de I,Atlas tellien oranais (O3) en analysant les principaux changements taxonomiques et chorologique. Les données de littérature disponibles ont été exploitées notamment (Quezel \& Santa, 1962-63; Vela \& Benhouhou, 2007) et analysés en utilisant l'index de la base de données de l'Afrique du Nord (Dobignard \& Chatelain, 2010-13). Cela a conduits à la suppression de 22 taxons de la liste de base comportant 94 taxons. Nous avons ensuite rajouté 15 nouveaux taxons signalés récemment dans la bibliographie au niveau de cette région ainsi que 3 taxons oubliés dans la première liste. La nouvelle liste de la flore endémique du 03 contenant 89 taxons a subi des changements considérables au niveau de sa composition floristique. Cette liste n'étant pas exhaustive devrait être enrichie par des nouveaux résultats au niveau des zones pas encore bien explorées sur le plan floristique notamment Mascara, Saida et Sidi Belabess.
\end{abstract}

Mots clés: Algérie, chorologie, endémique,liste, taxonomie.

\section{Abstract}

New data on endemic flora of the sub-sector of the Tellian Atlas Oranais "O3" (Western Algeria)

This study is a first regional synthesis aiming at proposing a new list of the endemic flora of the sub-sector of Oran Tell Atlas (O3), by analyzing the main taxonomic and chorological changes. The literature data available have been exploited in particular (Quézel \& Santa, 1962-63, VÉla \& Benhouhou, 2007) and analyzed using the index of the database of North Africa (Dobignard \& Chatelain, 2010-13). This led to the deletion of 22 taxa from the basic list of 94 taxa. We then added 15 new taxa reported recently in the bibliography at this region as well as 3 taxa forgotten in the first list. The new list of endemic flora of $\mathrm{O} 3$ containing 89 taxa has undergone considerable changes in its floristic composition. This list which is not exhaustive should be enriched by new results at the level of areas not yet well explored on the floristic plan including Mascara, Saida and Sidi Belabess.

Key words: Algeria, chorology, endemic, list, taxonomy.

\section{Introduction}

La région méditerranéenne se caractérise par une diversité biologique exceptionnelle ainsi qu'une richesse floristique estimée à 25000 espèces des plantes vasculaires, ce qui correspond à $9.2 \%$ de la flore mondiale sur un territoire représentant seulement $1.5 \%$ de la surface terrestre (Medail \& 
Quezel 1997; Myers 1988, 1990; Myers et al. 2000; Mittermeier et al. 2004)

L'Algérie, dont les ressources naturelles (faune, sol, végétation) ont fait l'objet de sollicitations précoces (Louni 1994) abrite des écosystèmes très diversifiés qui se caractérisent par une richesse floristique remarquable d'un intérêt mondial (Benabadji et al. 2007).

Les révisions taxonomiques récentes estiment le nombre d'espèces endémiques nationales pour le nord de l'Algérie (à l'exclusion du Sahara) à plus de 300 taxons (Yahi et al. 2012). Parmi les 406 endémiques du Maghreb (Algérie, Maroc, Tunisie), le secteur O3 sensu Quezel \& Santa (1962-63) se classe comme l'un des secteurs les plus riches en endémiques en Algérie avec 94 taxons (Vela \& Benhouhou 2007). Selon ces mêmes auteurs, plus de trois quart des taxons endémiques stricts d'Algérie ou sub-endémiques (77.9\%) sont des plantes plus ou moins rares dans le pays. Mise à part quelques rares études régionales très localisées sur le plan géographique récemment publiées (Hamel et al. 2013; Zedam 2014; Miara et al, 2017), la principale étude de référence sur les plantes endémiques et/ou rares en Algérie est celle de Vela \& Benhouhou (2007) qui est basée sur les données de la flore de Quezel \& Santa datant de (1962-63). Néanmoins, les données que contient cette flore ont subits beaucoup de changements taxonomiques, de nomenclature et de chorologie. Beaucoup de taxons ont soit changé de nomenclature (famille, genre, espèce, ...etc.), ou bien de chorologie (plantes auparavant endémiques découvertes dans les pays voisins ou en Europe, plantes nouvelles découvertes dans le pays, plantes endémiques auparavant cités dans des pays voisins désormais endémiques stricts du pays,....etc.). Cette étude aborde cette problématique au niveau du sous-secteur de l'Atlas tellien Oranais (O3) qui représente la plus grande portion de l'Atlas tellien occidental algérien.

L'objectif de cette étude est de détecter et mettre en exergue les changements ayant affecté la taxonomie (nomenclature) et la chorologie des taxons cités comme endémiques pour le secteur (O3) par Quezel \& Santa (1962-63). Nous tenterons par la suite de proposer une nouvelle liste actualisée des taxons endémiques du (O3).

\section{La zone d'etude}

Le sous-secteur de l'Atlas tellien oranais (O3) ou plutôt district atlasique Tiareti-Tlemcenien sensu Meddour (2010) représente la partie occidentale de l'Atlas Tellien algérien depuis les monts de Tiaret à l'est, de Frenda, de Saïda, de Daya jusqu'aux monts de Tlemcen à l'ouest. Le bioclimat prédominant est le semi-aride surtout dans sa variante d'hiver fraîche avec une pluviométrie annuelle variant de 350 à $650 \mathrm{~mm}$ ainsi qu>une période de sécheresse de 4 mois. Les monts de Tlemcen sont recouverts par quelques forêts de Chêne liège et même de Chêne zéen (Quercus faginea subsp. tlemcenensis), mais le Chêne vert est manifestement le plus répandu, ainsi que le $P$ in d'Alep et le Thuya de Berbérie (Meddour 2010). Sur les monts de dayas, de Saida et de Frenda c'est plutôt les formations de pin d'Alep (Pinus halepensis) qui dominent, alors que les monts de Tiaret sont recouverts par des groupements de chêne vert (Quercus ilex subsp. ballota) (Barry et al. 1974).

\section{Materiel et methodes}

Cette synthèse est essentiellement basée sur la comparaison des données bibliographiques (taxonomie et chorologie) des taxons figurant dans la liste des plantes endémiques du sous-secteur de l'Atlas tellien Oranais de Vela \& Benhouhou (2007) avec les données plus récentes notamment Dobignard et Chatelain (2010-13).

La liste de base a été extraite depuis la flore de Quezel \& Santa et contient 94 taxons endémiques relevant des catégories d'endémisme suivantes: endémique Algérie, Algérie-Maroc et AlgérieTunisie.

Les endémiques de l'Afrique du nord (Algérie, Maroc, Tunisie et Lybie) ne sont pas considérés ici afin de permettre une comparaison rigoureuses des résultats avec ceux de la liste de base.

Les analyses ont concerné essentiellement la nomenclature et la chorologie. Nous avons adopté la nomenclature des taxons de l'index de la base de données de l'Afrique du Nord (Dobignard \& Chatelain 2010-13). Concernant la chorologie des espèces, les données sont extraites des deux ouvrages (Quezel \& Santa 1962-63; Dobignard \& Chatelain 2010-13). Les analyses des données chorologiques ont concernés deux aspects principaux qui sont le statut et le type d'endémisme.

-Le statut d'endémisme: nous avons considéré sous cette notion la situation chorologique et biogéographique du taxon. C'est-à-dire que nous avons recherché si le taxon est endémique ou non sans tenir compte de sa catégorie d'endémisme.

-Le type d'endémisme: représente la catégorie d'endémisme dans laquelle le taxon endémique est affilié selon sa distribution géographique. C'està-dire sa présence ou non en Algérie et dans les pays voisins.

D'une manière générale, les données 
rassemblées ont servis à la construction d'une matrice de données qui a fait l'objet d'analyses suivant les étapes suivantes:

La recherche des changements de nomenclature en utilisant essentiellement la checklist des taxons endémiques de l'Afrique du Nord (EI Oualidi et al. 2012) ainsi que l'index synonymique de la Flore d'Afrique du Nord (Dobignard \& Chatelain 2010-13).

La révision des données chorologiques au niveau de cette liste en fonction de ces données. Cette révision concernant le statut et le type d'endémisme.

La suppression des taxons qui ne sont désormais plus endémiques après avoir changé de statut ou de type d'endémisme selon le cas.

L'ajout des taxons endémiques nouveaux cités récemment pour le sous-secteur (O3) au niveau des recherches et des travaux suivant: Meddour (2010); Babali (2014); Babali \& Bouazza (2016); Miara (2017).

La construction de la liste finale des taxons endémiques du $\mathrm{O} 3$.

\section{Resultats}

Au niveau du tableau 1, nous présentons la liste actualisée (nomenclature et chorologie) des plantes vasculaires endemiques du sous-secteur de l'Altlas tellien oranais.

\section{Nomenclature}

Les taxons ayant changé de nomenclature au niveau du genre, l'espèce ou la sous espèce sont du nombre de 27 taxons. Les taxons de rangs inferieurs tel que la variété n'ayant pas été considérés.

\section{Chorologie}

Nous avons constaté que 72 taxons parmi les 94 cités pour le (O3) ont conservé leurs statuts d'endémisme. C'est-à-dire que leurs type d'endémisme est soit resté stable ou bien a changé vers un autre type d'endémisme tout en restant endémique.

Parmi les taxons analysés, 12 taxons ont subi des changements au niveau de leurs statuts d'endémisme. Cela veut dire que ces taxons autrefois endémiques algériennes, Algérie Maroc ou Algérie-Tunisie (données de Quezel \& Santa 1962-63), ne sont désormais plus endémiques soit suite à leurs découvertes récentes dans des pays voisins avec une existence rejetée pour l'Algérie (cas de Genista hirsuta subsp. erioclada et Astragalus fontqueri Maire \& Sennen), soit suite à leurs découverte au niveau d'autres pays sud européens notamment en Espagne et en Italie alors que leurs présence en Algérie est toujours valide.

Ces taxons sont: Gagea mauritanica Durieu, Genista hirsuta subsp. erioclada (Spach) Raynaud, Hohenackeria polyodon Coss. \& Durieu, Limonium cymuliferum (Boiss.) Sauvage \& Vindt, Apteranthes europaea (Guss.) Murb. var. europaea, Filago micropodioides Lange, Gagea durieui Parl. ex Trab., Clypeola cyclodontea Delile, Genista tricuspidata Desf., Astragalus fontqueri Maire \& Sennen, Linum tenue Desf. subsp. tenue et Helianthemum polyanthum (Desf.) Pers.

Les taxons ayant conservé leurs types d'endémisme inchangés sont du nombre de 52 taxons (sans Silene imbricata -endémisme Maroc incertain). Parmi les 41 taxons endémiques stricts d'Algérie, seulement 14 taxons ont conservés leur type d'endémisme. Par contre, la majorité des taxons endémiques Algérie-Maroc sont restés stables.

Par ailleurs, les plantes ayant changés de type d'endémisme, c'est-à-dire ceux dont l'aire biogéographique connue a été modifié tout en restant endémiques à l,échelle de IsAfrique du Nord sont représentés par 31 taxons. Neuf endémiques algériennes ont été découvertes au Maroc ainsi que 7 autres signalés en Tunisie. Aussi, 3 taxons autrefois endémiques algériennes ou AlgérieMaroc sont désormais strictement marocains. Crest le cas des deux sous espèces Genista hirsuta subsp. erioclada (Spach) Raynaud» ainsi que Astragalus fontqueri.

\section{Les endémiques Afrique du Nord}

Au niveau de la liste de Vela \& Benhouhou (2007), les endémiques de l'Afrique du Nord ne sont pas prisent en considération. L'actualisation des données a montré que 10 taxons parmi les 94 endémiques du secteur sont désormais endémiques de l'Afrique du Nord. C'est-à-dire qu'ils sont présents dans au moins 3 pays de cette région. Ces taxons sont: Avena ventricosa Balansa ex Coss., Gagea granatellii (Parl.) Parl. subsp. granatellii, Spergularia munbyana Pomel, Filago fuscescens Pomel, Genista tricuspidata Desf. subsp. tricuspidata, Ferula longipes Coss. ex Bonnet \& Maury, Chiliadenus rupestris (Pomel) Brullo, Carthamus pectinatus Desf., Taraxacum microcephalum Pomel et Hedysarum pallidum Desf.

\section{Les taxons endémiques nouveaux pour le $\mathrm{O3}$}

Dans la présente étude, nous indiquons 15 taxons endémiques nouveaux pour le sous-secteur de I.Atlas tellien oranais (O3). Ces taxons ont été 
signalés dans plusieurs travaux récents à savoir: Meddour (2010) pour le sous-secteur (O3): Babali (2014) et Babali \& Bouazza (2016) pour la région de Tlemcen, ainsi que Miara (2017) pour la région de Tiaret.

A ces taxons, nous avons rajoutés 3 autres qui semblent avoir été oubliés dans la liste de Vela \& Benhouhou (2007). Il s'agit de: Adenocarpus faurei Maire, Nepeta algeriensis de Noé et Centaurea obtusiloba Batt.

\section{Nouvelle liste des plantes endémiques du 03}

A l'issu de ces analyses, nous proposons une nouvelle liste «actualisée» des plantes endémiques du sous-secteur de l'Atlas tellien Oranais (O3).

La nouvelle liste des plantes endemiques du O3 comporte désormais 89 taxons suite à une profonde modification. En effet, méme si le nombre des taxons n'a pas été significativement modifié par rapport à la premiere liste comportant 94 taxons, les changements taxonomiques et chorologiques ont été considerables, avec 18 taxons rajoutés (15 nouveaux et 3 oubliés), et 22 taxons supprimés (12 taxons ayant changé de statut d'endémisme et 10 ayant devenus endémiques de l'Afrique du nord).

Notons le cas de Genista hirsuta qui est représenté dans la liste de base par 2 taxons (subsp. erioclada et atlantica). Ces deux taxons sont rassemblés après l'actualisation en un seul taxon Genista hirsuta subsp. erioclada. Donc la nouvelle liste contenait d'emblais 93 taxons et cela avant de procéder à la suppression des taxons ayant changés de statut d'endémismes ou ceux devenus endémiques nord africains.

\section{Discussion}

L'actualisation des données taxonomiques et de nomenclature a permis de mettre en évidence beaucoup de changements qui ont affectés la flore du secteur O3. Malgré la disponibilité des sources bibliographiques, nous avons toutefois remarqué une certaine négligence de cet aspect dans plusieurs recherches publiée récemment en Algérie (Babali 2014; Zedam 2015). Ces aspects possédant un intérêt majeur notamment pour les taxonomistes et botanistes sont a considéré en priorités dans les recherches scientifiques (RAO, 2004).

Concernant le statut d'endémisme des espèces, nous avons remarqué que Genista hirsuta subsp. erioclada (Spach) Raynau et Astragalus fontqueri Maire \& Sennen. autrefois endémiques Algérie et Algérie-Maroc respectivement, ne figurent pas dans la liste des endémiques du Maroc de Rankou et al. (2013). Aussi, Genista hirsuta subsp. erioclada ne figure pas dans la liste d'El Oualidi et al. (2012). Ainsi, la révision de la liste des endémiques du Maroc semble nécessaire à la lumière des changements récents.

Concernant le type d'endémisme, cet aspect semble assez négligé par les chercheurs algériens. Plusieurs auteurs semblent avoir des difficultés relatives à la mise à jour des données chorologiques de leurs plantes. Si l'on prend l'exemple d'une des études les plus récentes, celle de Zedam (2015) est la plus représentative. En effet, cet auteur accumule plusieurs erreurs en rapport avec la distribution géographique des taxons endémiques mentionnés dans son étude. Csest le cas pour Silene arenarioides Desf. qui est mentionnée comme endémique Algériennes alors qu'elle est plutôt Nord-Africaine (Algérie-MarocTunisie). De même pour Herniaria mauritanica Murb. et Limonium cymuliferum (Boiss.) Sauvage \& Vindt qui sont cités comme endémiques Algériennes strictes alors qu'elles sont désormais Algérie-Tunisie et Algérie-Maroc respectivement. Aussi, Hedysarum carnosum Desf. est cité a tort comme endémique Algérie-Tunisie. Ce taxon est plutôt endémique de l'Afrique du Nord.

Concernant les endémiques de l'Afrique du Nord non retenus dans la présente étude, il s'agit plutôt drun endémisme qui nıest pas souvent considéré à cause de liétendue de lsaire de répartition de ces taxons qui sont généralement présents dans 3 pays ou plus (Vela \& Benhouhou 2007). Leurs nombre qui n'a pas été considéré pour la liste finale des endémiques du O3, aurait pu augmenter significativement le nombre des taxons endémiques du secteur étudié. En plus des 10 taxons ressortis suite à lsactualisation des données, au moins une autre dizaine figurant dans les travaux de Meddour (2010), Babali (2014), Babali \& Bouazza (2016) et Miara (2017) aurait pu être rajoutée à la liste finale.

Le nombre des taxons nouveaux signalés pour la région semble assez faible et ne reflétant pas la réalité. Cela pourra être justifié par la rareté des études et des inventaires floristiques réalisés dans ce secteur. A lsimage de tout le territoire algérien, le sous-secteur $\mathrm{O} 3$ reste une région très mal explorée et besoin de plus diattention notamment en termes de richesse en endémiques (Miara 2017). C'est le cas notamment de la région du nord de Saida ainsi que le sud de Mascara et de Sidi Belabess ou aucune étude d'inventaire floristique n'a été entreprise depuis l'indépendance. Les recherches devront aussi s'intensifié au niveau des confins algéro-marocains (la région de Tlemcen) ou beaucoup de taxons marocains sont succeptibles d'exister à travers le prolongement naturelle de 


\begin{tabular}{|c|c|}
\hline Taxons & Chorologie \\
\hline $\begin{array}{c}\text { Adenocarpus faurei Maire } \\
\text { Ammochloa pungens (Schreb.) Boiss. }\end{array}$ & Alg Alg-Mar \\
\hline Armeria ebracteata Pomel & Alg-Mar \\
\hline Bellis rotundifolia (Desf.) Boiss. \& Reut. & Alg-Mar \\
\hline Bupleurum montanum Coss. & Alg-Mar \\
\hline Campanula velata Pomel subsp. velata & Alg-Mar \\
\hline Cardopatium amethystinum Spach & Alg-Tun \\
\hline Carlina atlantica Pomel & Alg-Mar \\
\hline Carlina macrophylla (Desf.) DC. & Alg-Mar \\
\hline Carthamus carthamoides (Pomel) Batt. & Alg-Mar \\
\hline Centaurea fragilis Durieu & Alg-Mar \\
\hline Centaurea infestans Durieu & Alg-Mar \\
\hline Centaurea nana Desf. & Alg-Mar \\
\hline Centaurea obtusiloba Batt. & Alg \\
\hline Centaurea oranensis Greuter \& M.V. Agab. & Alg-Mar \\
\hline Centaurea parviflora Desf. & Alg-Tun \\
\hline Centaurea phaeolepis Coss. & Alg \\
\hline Centranthus battandieri Maire & Alg-Mar \\
\hline Cistus munbyi Pomel & Alg-Mar \\
\hline Coleostephus multicaulis (Desf.) Durieu & Alg \\
\hline Cordylocarpus muricatus Desf. & Alg-Mar \\
\hline $\begin{array}{c}\text { Crepis arenaria (Pomel) Pomel } \\
\text { subsp. arenaria }\end{array}$ & Alg \\
\hline Cynoglossum mathezii Greuter \& Burdet & Alg-Mar \\
\hline Cynosurus elegans Desf. subsp. elegans & Alg-Tun \\
\hline $\begin{array}{l}\text { Dactylorhiza durandii } \\
\text { (Boiss. \& Reut.) M. Laínz }\end{array}$ & Alg-Mar \\
\hline Delphinium mauritanicum Coss. & Alg-Mar \\
\hline $\begin{array}{l}\text { Dichoropetalum munbyi (Boiss.) } \\
\text { Pimenov \& Kljuykov }\end{array}$ & Alg-Mar \\
\hline Erodium medeense Batt. & Alg \\
\hline Erodium populifolium L'Hér. & Alg-Tun \\
\hline $\begin{array}{l}\text { Erodium tordylioides (Desf.) } \\
\text { L'Hér. subsp. tordylioides }\end{array}$ & Alg-Mar \\
\hline $\begin{array}{l}\text { Euphorbia bupleuroides Desf. } \\
\text { subsp. bupleuroides }\end{array}$ & Alg -/Mar? \\
\hline Festuca algeriensis Trab. & Alg \\
\hline Filago congesta Guss. ex DC. & Alg-Mar \\
\hline Filago crocidion (Pomel) Chrtek \& Holub & Alg-Mar \\
\hline Filago duriaei Lange & Alg-Mar \\
\hline Gagea algeriensis Chabert & Alg-Mar \\
\hline Gagea chabertii A. Terracc. & Alg-Tun \\
\hline Galium bourgaeanum Coss. ex Ball & Alg-Mar \\
\hline Genista cephalantha Spach. & Alg-Mar \\
\hline
\end{tabular}

\begin{tabular}{|c|c|}
\hline Taxons & Chorologie \\
\hline Genista quadriflora Munby & Alg-Mar \\
\hline $\begin{array}{c}\text { Guenthera setulosa (Boiss. \& Reut.) } \\
\text { Gómez-Campo }\end{array}$ & Alg-Mar \\
\hline Helminthotheca glomerata (Pomel) Greuter & Alg-Mar \\
\hline Iris tingitana Boiss. \& Reut. & Alg-Mar \\
\hline $\begin{array}{l}\text { Lamium amplexicaule } \\
\text { subsp. mauritanicum (Gand. ex Batt.) Maire }\end{array}$ & Alg-Mar \\
\hline Leontodon balansae Boiss. & Alg-Mar \\
\hline Lepidium violaceum (Munby) Al-Shehbaz & Alg-Mar \\
\hline Linaria latifolia Desf. & Alg-Mar \\
\hline Linaria tristis subsp. marginata (Desf.) Maire & Alg \\
\hline $\begin{array}{l}\text { Linum corymbiferum Desf. } \\
\text { subsp. corymbiferum }\end{array}$ & Alg-Tun \\
\hline Linum grandiflorum Desf. & Alg-Tun \\
\hline $\begin{array}{l}\text { Linum tenue subsp. munbyanum } \\
\text { (Boiss. \& Reut.) Batt. }\end{array}$ & Alg-Mar \\
\hline Malcolmia arenaria (Desf.) DC. & Alg-Mar \\
\hline Marrubium alyssoides Pomel & Alg-Mar \\
\hline Melilotus macrocarpus Coss. \& Durieu & Alg-Tun \\
\hline Micromeria debilis Pomel & Alg-Mar \\
\hline Micromeria hochreutineri (Briq.) Maire & Alg-Mar \\
\hline Nepeta algeriensis de Noé & Alg \\
\hline $\begin{array}{l}\text { Ononis antennata subsp. massesylia } \\
\text { (Pomel) Sirj. }\end{array}$ & Alg-Mar \\
\hline Ononis avellana Pomel & Alg \\
\hline Ononis crinita Pomel & Alg \\
\hline Ononis rosea Durieu & Alg-Tun \\
\hline Ononis serotina Pomel & Alg \\
\hline $\begin{array}{c}\text { Origanum vulgare subsp. glandulosum } \\
\text { (Desf.) letsw. }\end{array}$ & Alg-Tun \\
\hline Plagius grandis (L.) Alavi \& Heywood & Alg-Tun \\
\hline Plantago mauritanica Boiss. \& Reut. & Alg-Mar \\
\hline Polygala rosea Desf. & Alg \\
\hline $\begin{array}{l}\text { Pulicaria vulgaris subsp. pomeliana } \\
\text { (Faure \& Maire) E. Gamal-Eldin }\end{array}$ & Alg \\
\hline Romulea numidica Jord. \& Fourr & Alg-Mar \\
\hline Salvia algeriensis Desf. & Alg-Mar \\
\hline Sideritis guyoniana Boiss. \& Reut. & Alg-Mar \\
\hline Silene argillosa Munby & Alg-Mar \\
\hline Silene cinerea Desf. & Alg-Mar \\
\hline Silene glaberrima Faure \& Maire & Alg \\
\hline Silene imbricata Desf. & Alg/ Mar? \\
\hline Silene velutinoides Pomel & Alg-Mar \\
\hline Stachys duriaei de Noé & Alg-Tun \\
\hline
\end{tabular}

Tableau 1. Continuation à la page suivante. 


\begin{tabular}{|c|c|}
\hline Taxons & Chorologie \\
\hline Stellarioides sessiliflora (Desf.) Speta & Alg-Mar \\
Stoibrax pomelianum (Maire) B.L. Burtt & Alg-Mar \\
Thalictrum speciosissimum L. & Alg-Mar \\
Thymus guyonii de Noé & Alg \\
Thymus lanceolatus Desf. & Alg \\
Thymus munbyanus Boiss. \& Reut. & Alg-Mar \\
subsp. munbyanus & Alg-Mar \\
Thymus munbyanus subsp. coloratus \\
(Boiss. \& Reut.) Greuter \& Burdet
\end{tabular}

\begin{tabular}{|c|c|}
\hline Taxons & Chorologie \\
\hline Thymus pallescens de Noé & Alg-Mar \\
Trisetaria nitida (Desf.) Maire & Alg \\
Valerianella leptocarpa Pomel & Alg \\
Verbascum betonicifolium (Desf.) Kuntze & Alg \\
Verbascum faurei (Murb.) Hub.-Mor. & Alg-Mar \\
Verbascum maurum Maire \& Murb. & Alg-Mar \\
\hline
\end{tabular}

Tableau 1. Nouvelle liste des plantes endémiques du secteur O3: La nomenclature est selon Dobignard \& Chatelain (2010-13).

Table 1. New list of endemic plants in sector O3: The nomenclature is according to Dobignard \& Chatelain (2010-13).

la chaine atlasique. Récemment, Sekkal et al. (2018) ont découvert 3 nouveaux taxons (Echium modestum Ball, Plantago benisnassenii Romo, Peris \& Stübing, Teucrium doumerguei Sennen) dans les Traras près des frontières marocaines. Egalement, Hamel \& Boulemtafes (2017) ont découvert une endémique tyrrhénienne Soleirolia soleirolii (Req.) Dandy à Annaba près des frontières Tunisiennes.

Globalement, il convient de souligner que sur les plans nomenclature et chorologie, les changements ayant subite la flore d'Algérie ainsi que celles des pays voisins suite aux révisions taxonomiques récentes ont été synthétisé au niveau de l'index de la flore de l'Afrique du Nord de Dobignard \& chatelain (2010-13).

II faudra ainsi insister sur l'importance de cet ouvrage qui constitue actuellement le meilleur repère et source d'information sur la flore d'Algérie et des pays voisins. Toutefois, les connaissances ne sont toujours pas complètes et beaucoup d'incertitudes sont notés pour un bon nombre de taxons notamment sur le plan de la distribution géographique.

Bien que depuis la publication de cet index, beaucoup de chercheurs et auteurs tiennent compte de ces changements grâce à ces données ainsi que d'autres données disponibles sur internet (référentiel TAXREF, the plant list,...etc.), nous avons toutefois remarqué quelques cas de non prise en considération des aspects nomenclaturaux cruciaux dans toute recherche scientifique portant sur l'inventaire et la caractérisation de la phytodiversité végétale. Babali (2014) par exemple néglige l'actualisation de la nomenclature au niveau de son document notamment au niveau de quelques familles anciennes adoptées. Des taxons comme Fritillaria messanensis appartenant désormais aux Hyacinthaceae a été rangé dans les Lilaceae. Aussi, il utilise toujours le nom d'Anagallis arvensis L. pour Lysimachia arvensis (L.) U. Manns \& Anderb. Aussi, Zedam (2015) cite Astragalus fontanesii subsp. tragacanthoides (Desf.) Maire qui est un taxon rejeté et est synonyme d'Astragalus armatus Willd. subsp. armatus. Ces aspects sont également négligés au niveau des recherches ethnobotaniques ou plusieurs anomalies et erreurs ont été relevées au niveau de certaines études publiées (Miara et al. 2018).

Sur le plan chorologique, il en est de même au niveau de plusieurs études analysées. Lors de l,analyse des quelques rares inventaires portant sur les endémiques en Algérie, nous avons constaté que cet aspect est souvent oubliés par les auteurs qui présentent les taxons sous leurs statuts chorologique anciens. C>est le cas dans létude de Zedam (2014) qui cite par exemple Frankenia thymifolia Desf. comme une endémique de l'Afrique du Nord, alors qu'elle figure depuis longtemps dans Flora Iberica (3: 451, 1993).

En générale, les auteurs sous estiment cet aspect et ne procèdent souvent pas à la vérification de la situation chorologique des taxons mentionnés dans leurs publications. C'est le cas par exemple de Babali (2014) qui cite Fedia cornucopiae pour l'Algérie alors que cette espèce est plutôt rejetée pour ce pays.

\section{Conclusion}

Cette première synthèse sur les plantes endémiques du sous-secteur de l'Atlas tellien oranais (O3) a permis d'abord de mettre en exergue les changements qui ont affecté les principaux aspects de cette flore (nomenclature, chorologie: statut et type d'endémisme), puis de proposer une 
nouvelle liste de cette flore régionale en se basant sur les données récemment publiées pour ce secteur biogéographique.

Cette liste ayant subi des changements assez importants dans sa composition, n'a pas connue un changement significatif sur le plan numérique.

Beaucoup d'espèce autrefois endémiques algériennes sont devenue algéro-marocaines ou algéro-tunisienne ou encore strictement marocaines. Cela dénote d'un gros travail de recherche qui a été réalisé dans les pays voisins contre des rares travaux en Algérie notamment sur les confins algéro-marocains ou beaucoup d'espèces actuellement marocaines peuvent également se retrouver sur le territoire algérien.

Ainsi, beaucoup de travail reste à faire sur le plan de l>exploration floristique à lıéchelle du pays notamment à l>ouest. Des zones comme Mascara, Saida et Sidi Belabess devraient bénéficier de plus d)intérêt scientifique et de recherches exploratoires.

\section{Remerciements}

L'auteur tiens à remercier Dr. Errol Véla (Université de Montpellier) d'avoir eu la gentillesse de fournir la liste des plantes endémiques du secteur $\mathrm{O} 3$ sur laquelle cette étude s'est basée.

\section{Bibliographie}

BabaliB. (2014). Contributionàuneétude phytoécologique des monts de Moutas (Tlemcen- Algérie occidentale): Aspects syntaxonomique, biogéographique et dynamique. Thése Doct. Université de Tlemcen. 160p.

Babali B. \& Bouazza M. (2016). Redécouverte de Pulicaria vulgaris subsp. pomeliana (Faure et Maire) E. Gamal-Eldin (Asteraceae) dans la région de Tlemcen (Algérie occidentale). Bull Soc Lin Prov. 67, 45-52.

Barry J.P., Celles J.C. \& Faurell L. (1974). Carte internationale du tapis végétal et des conditions écologiques. Feuille d'Alger au 1/1000.000. Bull. Soc. Hist. Nat. Afr. Nord. Alger.

Benabadji N., Benmansour D. \& Bouazza M. (2007). La flore des monts d'Ain Fezza dans l'ouest algerien, biodiversite et dynamique. Sciences \& Technologie. $26: 47-59$.

Dobignard A. \& Chatelain C. (2010-2011-2012 et 2013). Index synonymique de la Flore d'Afrique du Nord. Conservatoire et Jardin botaniques de la Ville de Genève $(\mathrm{CH}), 5$ Vol.

El Oualidi J., Khamar H., Fennane M., Ibn Tattou M., Chauvet S. \& Taleb M.S. (2012). Checklist des endémiques et spécimens types de la flore vasculaire de l'Afrique du Nord. Doc Inst Sci. Universite mohammed v-agdal, Rabat. 25,192 p.

Hamel T., Seridi R., De Belair G., Slimani A. \& Babali B. (2013). Flore vasculaire rare et endémique de la péninsule de l'Edough (Nord-Est algérien). Rev. Sci.
Technol. Synthèse. 26, 65-74.

Hamel T. \& Boulemtafes, A. (2017). Découverte d'une endémique tyrrhénienne Soleirolia soleirolii (Urticaceae) en Algérie (Afrique du Nord). Fl. Medit. 27: 185-193.

Louni, D. (1994). Les Forêts algériennes. Forêts méditerranéennes. T: XV, N 7, 59-63.

Médail F. \& Quézel, P. (1997). Hot-spots analysis fo $r$ conservation of plant biodiversity in the Mediterranean basin. Ann. Missouri Bot. Gard. 84: 112-127.

Meddour R. (2010). Bioclimatologie, Phytogéographie et Phytosociologie en Algérie. Exemple des groupements forestiers et pré forestiers de la Kabylie Djurdjurieenne. Thèse doct d'état. Univ Tizi Ouzou. 397 p.

Miara M-D. (2017). Analyse floristique et structure de la végétation de la région de Tiaret. Thése Doct. Univ Oran. 162p.

Miara M-D., Bendif H., Rebbas K., Bounar R., Ait Hammou M. \& Maggi F., (2018). Medicinal plants and their traditional uses in the highlands region of Bordj Bou Arreridj (Northeast Algeria). Journal of Herbal Medicine. Soumis.

Mittermeier R-A., Robles Gil P., Hoffmann M., Pilgrim J., Brooks T., Mittermeier C-G., Lamoreux J. \& Da Fonseca, G-A-B. (2004). Hotspots revisited: Earth's biologically richest and most endangered terrestrial ecoregions, Preface by Peter A. Seligmann, Foreword by Harrison Ford. Cemex/Conservation International/ Agrupacion, Sierra Madre/Monterrey/Mexico, p. 392.

Myers, N. (1988). Threatened biotas: Hotspots in tropical forests. Environmentalist. 8, 178-208.

Myers, N. (1990). The biodiversity challenge: Expanded hotspots analysis. Environmentalist. 10, 243-256. Myers N., Mittermeier R-A., Mittermeier C-G., Da Fonseca G-A-B. \& Kent J. (2000). Biodiversity hotspots for conservation priorities. Nature. 403, 853-858.

Quezel P. \& Santa S., (1962-1963). Nouvelle flore de l'Algérie et des régions désertiques méridionales. In CNRS (Ed). Paris, 2 vols, $1170 \mathrm{p}$

Rankou H., Culham A., Jury S.L. \& Christenhusz M. (2013). The Endemic Flora of Morocco. Phytotaxa. 78 (1): 1-69

Rao M-K. (2004). The importance of botanical nomenclature and synonymy in taxonomy and biodiversity M. K. Current Science. Vol. 87, No. 5, 602-606.

Sekkal F.Z., Hadjadj-Aoul S. \& Véla E. (2018). Echium modestum ball, Plantago benisnassenii Romo, Peris \& stübing, Teucrium doumerguei sennen : nouveaux taxons pour la flore d'Algérie (massif des Traras). Revu d'écologie (terre et vie). Vol. 73 (1), 41-56

Vela E. \& Benhouhou S. (2007). Evaluation d'un nouveau point chaud de biodiversité végétale dans le bassin méditerranéen (Afrique du nord). C.R. Biologies. 330: 589-605.

Yahi N., Vela E., Benhouhou S., De Belair G. \& Gharzouli R. (2012). Identifying Important Plants Areas (Key Biodiversity Areas for Plants) in northern Algeria. Journal of Threatened Taxa. 4 (8) : 2753-2765.

Zedam, A. (2015). Etude de la flore endémique de la zone humide du Chott El Hodna, inventaire et préservation. Thèse Doct, Université de Sétif, Algérie, 368p. 